\title{
Transverse momentum-dependent parton distribution functions in lattice QCD
}

\author{
M. Engelhardt ${ }^{*}{ }^{\dagger}$, B. Musch ${ }^{b}$, P. Hägler ${ }^{c}$, J. Negele ${ }^{d}$, and A. Schäfer \\ ${ }^{a}$ Department of Physics, New Mexico State University, Las Cruces, NM 88003, USA \\ ${ }^{b}$ Theory Center, Jefferson National Laboratory, Newport News, VA 23606, USA \\ ${ }^{c}$ Institut für Kernphysik, Johannes Gutenberg-Universität Mainz, 55128 Mainz, Germany \\ ${ }^{d}$ Center for Theoretical Physics, Massachusetts Institute of Technology, Cambridge, MA 02139, \\ USA \\ ${ }^{e}$ Institut für Theoretische Physik, Universität Regensburg, 93040 Regensburg, Germany \\ ${ }^{\dagger} E$-mail: engel@nmsu.edu
}

\begin{abstract}
A fundamental structural property of the nucleon is the distribution of quark momenta, both parallel as well as perpendicular to its propagation. Experimentally, this information is accessible via selected processes such as semi-inclusive deep inelastic scattering (SIDIS) and the Drell-Yan process (DY), which can be parametrized in terms of transverse momentum-dependent parton distributions (TMDs). On the other hand, these distribution functions can be extracted from nucleon matrix elements of a certain class of bilocal quark operators in which the quarks are connected by a staple-shaped Wilson line serving to incorporate initial state (DY) or final state (SIDIS) interactions. A scheme for evaluating such matrix elements within lattice QCD is developed. This requires casting the calculation in a particular Lorentz frame, which is facilitated by a parametrization of the matrix elements in terms of invariant amplitudes. Exploratory results are presented for the time-reversal odd Sivers and Boer-Mulders transverse momentum shifts.
\end{abstract}

The 7th International Workshop on Chiral Dynamics

August 6-10, 2012

Jefferson Lab, Newport News, Virginia, USA

${ }^{*}$ Speaker. 


\section{Introduction}

In the description of nucleon structure, transverse momentum-dependent parton distribution functions [1] (TMDs) play a role complementary to generalized parton distributions (GPDs). Whereas GPDs encode information about the transverse spatial distribution of partons (through Fourier transformation with respect to the momentum transfer), TMDs contain information about the transverse momentum distribution of partons. As detailed further below, the definition of TMDs involves a number of subtleties not encountered in the case of GPDs, which also must be taken into account in formulating corresponding lattice QCD calculational schemes. Cast in a Lorentz frame in which the nucleon of mass $m_{N}$ propagates with a large momentum in 3-direction, $P^{+} \equiv\left(P^{0}+P^{3}\right) / \sqrt{2} \gg m_{N}$, the quark momentum components scale such that TMDs are principally functions $f\left(x, k_{T}\right)$ of the quark longitudinal momentum fraction $x=k^{+} / P^{+}$and the quark transverse momentum vector $k_{T}$, with the dependence on the component $k^{-} \equiv\left(k^{0}-k^{3}\right) / \sqrt{2} \ll m_{N}$ becoming ignorable in this limit. $f\left(x, k_{T}\right)$ will thus be regarded as having been integrated over $k^{-}$. The TMDs also depend on a collection of further parameters which will be specified below as needed.

Experimentally, TMDs manifest themselves in angular asymmetries observed in processes such as semi-inclusive deep inelastic scattering (SIDIS) and the Drell-Yan (DY) process. Corresponding signatures have emerged at COMPASS, HERMES and JLab [2-4], and that has motivated targeting a significant part of the physics program at future experiments in this direction, e.g., at the upgraded JLab $12 \mathrm{GeV}$ facility and at the proposed electron-ion collider (EIC). To relate the experimental signature to the nucleon structure encoded in TMDs, a suitable factorization framework is required. One possible such framework which is particularly well-suited for connecting phenomenology to a lattice QCD calculation has been advanced in [5-8]. Factorization in the TMD context is considerably more involved than standard collinear factorization, with the resulting TMDs in general being process-dependent, via initial and/or final state interactions between the struck quark and the nucleon remnant.

The main thrust of the present work lies in casting the phenomenological definition of TMDs into a form amenable to evaluation within lattice QCD, and presenting exploratory results for selected TMD observables. This is facilitated by writing the fundamental TMD correlator introduced below in terms of invariant amplitudes, so that the problem can be transformed to a Lorentz frame in which rotation to Euclidean lattice time becomes simple. In particular, time-reversal odd (T-odd) observables such as the Sivers and Boer-Mulders shifts will be discussed. A detailed account of this work was presented in [9].

\section{Definition of TMD observables}

The fundamental correlator defining TMDs is of the form

$$
\Phi^{[\Gamma]}\left(x, k_{T}, P, S, \ldots\right)=\left.\int \frac{d^{2} b_{T}}{(2 \pi)^{2}} \int \frac{d(b \cdot P)}{(2 \pi) P^{+}} \exp \left(i x(b \cdot P)-i b_{T} \cdot k_{T}\right) \frac{\widetilde{\Phi}_{\text {unsubtr. }}^{[\Gamma]}(b, P, S, \ldots)}{\widetilde{\mathscr{S}}\left(b^{2}, \ldots\right)}\right|_{b^{+}=0}
$$

with

$$
\widetilde{\Phi}_{\text {unsubtr. }}^{[\Gamma]}(b, P, S, \ldots) \equiv \frac{1}{2}\langle P, S|\bar{q}(0) \Gamma \mathscr{U}[0, \ldots, b] q(b)| P, S\rangle
$$


where $S$ denotes the spin of the nucleon and $\Gamma$ stands for an arbitrary $\gamma$-matrix structure. Heuristically, the Fourier-transformed bilocal quark bilinear operator counts quarks of momentum $k$ in the nucleon state, with $\Gamma$ controlling the specific spinor components involved. However, gauge invariance additionally enforces the introduction of the gauge connection $\mathscr{U}$, the precise path of which is not specified at this point; its choice will be guided by the physical process under consideration. In turn, the presence of $\mathscr{U}$ introduces divergences additional to the wave function renormalizations of the quark operators (this is indicated by the subscript "unsubtr."); these divergences accordingly must be compensated by the additional "soft factor" $\widetilde{\mathscr{S}}$. Here, $\widetilde{\mathscr{S}}$ does not need to be specified in detail, since only appropriate ratios in which the soft factors cancel will ultimately be considered. Finally, $\Phi^{[\Gamma]}\left(x, k_{T}, P, S, \ldots\right)$ is, as noted further above, a function only of the three quark momentum components contained in $x$ and $k_{T}$, whereas the small component $k^{-}$is integrated over; thus, in its Fourier transform, the conjugate component $b^{+}$is set to zero, as written in (2.1). The pairs $x \leftrightarrow(b \cdot P)$ and $k_{T} \leftrightarrow b_{T}$ consequently act as pairs of Fourier conjugate variables in (2.1). It is important to note for further reference that the quark separation $b$ in general includes a transverse component $b_{T}$ and therefore is generically space-like.

Decomposing the correlator $\Phi^{[\Gamma]}\left(x, k_{T}, P, S, \ldots\right)$ into the relevant Lorentz structures yields the TMDs as coefficient functions. At leading twist,

$$
\begin{aligned}
\Phi^{\left[\gamma^{+}\right]} & =f_{1}-\left[\frac{\varepsilon_{i j} k_{i} S_{j}}{m_{N}} f_{1 T}^{\perp}\right]_{\text {odd }} \\
\Phi^{\left[\gamma^{+} \gamma^{5}\right]} & =\Lambda g_{1}+\frac{k_{T} \cdot S_{T}}{m_{N}} g_{1 T} \\
\Phi^{\left[i \sigma^{i+} \gamma^{5}\right]} & =S_{i} h_{1}+\frac{\left(2 k_{i} k_{j}-k_{T}^{2} \delta_{i j}\right) S_{j}}{2 m_{N}^{2}} h_{1 T}^{\perp}+\frac{\Lambda k_{i}}{m_{N}} h_{1 L}^{\perp}+\left[\frac{\varepsilon_{i j} k_{j}}{m_{N}} h_{1}^{\perp}\right]_{\text {odd }}
\end{aligned}
$$

where $\Lambda$ denotes the nucleon helicity (i.e., $S^{+}=\Lambda P^{+} / m_{N}, S^{-}=-\Lambda m_{N} / 2 P^{+}$). In particular, the two TMDs $f_{1 T}^{\perp}$ and $h_{1}^{\perp}$ are odd under time reversal. Nonvanishing effects in these channels can only occur if a mechanism is operative which breaks time-reversal invariance. The former TMD, characterizing the unpolarized distribution of quarks in a transversely polarized nucleon, is the Sivers function, whereas the latter TMD, characterizing the distribution of transversely polarized quarks in an unpolarized nucleon, is the Boer-Mulders function.

Up to this point, no reference has been made to a physical process which may be parametrized by the TMDs. However, the usefulness of a definition of TMDs is contingent upon such a connection being possible. This requires a factorization framework which allows one to separate the description of the physical process into the hard, perturbative vertex, a TMD encoding the structure of the nucleon, and further components such as fragmentation functions describing the hadronization of the struck quark. In general, the possibility of a factorization of this kind is not guaranteed. For example, for reaction classes with multiple hadrons in both the initial and the final state, it has been argued that TMD factorization in general fails [10]. However, for certain processes, including semi-inclusive deep inelastic scattering (SIDIS) and the Drell-Yan (DY) process, factorization arguments have indeed been constructed, one possible approach having been advanced, e.g., in [5-8]. Fig. 1 schematically exhibits the principal elements involved in a description of SIDIS. One particularly noteworthy aspect is the final-state gluon exchanges between the struck quark and the nucleon remnant. These final state effects break time-reversal invariance and thus lead to nontriv- 


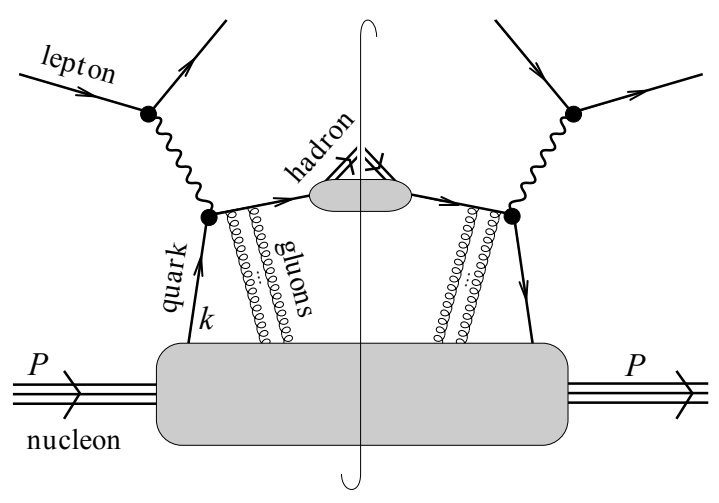

Figure 1: Illustration of the elements of SIDIS factorization. The lower shaded bubble represents the structure parametrized by TMDs.

ial T-odd TMDs. At a formal level, a resummation of these gluon exchanges in the spirit of an eikonal approximation yields a Wilson line approximately following the trajectory of the struck quark, close to the light cone. This motivates a specific choice for the gauge connection between the quark operators in (2.2). Namely, parallel Wilson lines are attached to both of the quark operators, extending to large distances along a direction $v$ close to the light cone; at the far end, these lines are connected by a Wilson line in the $b$ direction to maintain gauge invariance. The result is a staple-shaped connection $\mathscr{U}[0, \eta v, \eta v+b, b]$, cf. Fig. 2, where the path links the positions in the argument of $\mathscr{U}$ with straight line segments, and $\eta$ parametrizes the length of the staple. Formally, thus, it is the introduction of the additional vector $v$ which breaks the symmetry under time reversal and makes nonvanishing Sivers and Boer-Mulders effects possible.

At first sight, the most convenient choice for the staple direction $v$ would seem to be a light-like vector. However, beyond tree level, this introduces rapidity divergences which require regularization. One advantageous way to accomplish this is to take $v$ slightly off the light cone into the space-like region [5,6], with perturbative evolution equations governing the approach to the light cone [7]. Within this scheme, a "modified universality" has been established, i.e., common TMDs describing both SIDIS and DY, except that in the DY process, it is initial state interactions which play a crucial role; correspondingly, the staple direction $v$ is inverted and the T-odd TMDs acquire a minus sign. A scheme in which $v$ (along with the quark operator separation $b$ ) is generically spacelike is also attractive from the point of view of lattice QCD, as discussed further below. It will

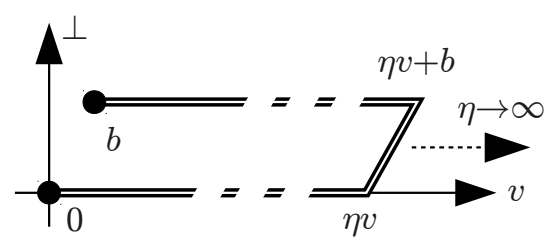

Figure 2: Staple-shaped gauge connection $\mathscr{U}[0, \eta v, \eta v+b, b]$. 
thus constitute the starting point for the development of the lattice calculation. A useful parameter characterizing how close $v$ is to the light cone is the Collins-Soper evolution parameter

$$
\hat{\zeta}=\frac{v \cdot P}{|v||P|},
$$

in terms of which the light cone is approached for $\hat{\zeta} \rightarrow \infty$.

The correlator (2.2) can be decomposed in terms of invariant amplitudes $\widetilde{A}_{i B}$. Listing only the leading twist components,

$$
\begin{aligned}
& \frac{1}{2 P^{+}} \widetilde{\Phi}_{\text {unsubtr. }}^{\left[\gamma^{+}\right]}=\widetilde{A}_{2 B}+i m_{N} \varepsilon_{i j} b_{i} S_{j} \widetilde{A}_{12 B} \\
& \frac{1}{2 P^{+}} \widetilde{\Phi}_{\text {unsubtr. }}^{\left[\gamma^{+} \gamma^{5}\right]}=-\Lambda \widetilde{A}_{6 B}+i\left[(b \cdot P) \Lambda-m_{N}\left(b_{T} \cdot S_{T}\right)\right] \widetilde{A}_{7 B} \\
& \frac{1}{2 P^{+}} \widetilde{\Phi}_{\text {unsubtr. }}^{\left[i \sigma^{i+} \gamma^{5}\right]}=i m_{N} \varepsilon_{i j} b_{j} \widetilde{A}_{4 B}-S_{i} \widetilde{A}_{9 B}-i m_{N} \Lambda b_{i} \widetilde{A}_{10 B}+m_{N}\left[(b \cdot P) \Lambda-m_{N}\left(b_{T} \cdot S_{T}\right)\right] b_{i} \widetilde{A}_{11 B} .
\end{aligned}
$$

These amplitudes are useful in that they can be evaluated in any desired Lorentz frame; they will thus facilitate casting the problem in a frame which is particularly suited for the lattice calculation. On the other hand, in view of (2.3)-(2.5), they are clearly closely related to Fourier-transformed TMDs. Performing the corresponding algebra, and quoting only the components necessary for defining the Sivers and Boer-Mulders shifts below,

$$
\begin{aligned}
\tilde{f}_{1}^{[1](0)}\left(b_{T}^{2}, \hat{\zeta}, \ldots, \eta v \cdot P\right) & =2 \widetilde{A}_{2 B}\left(-b_{T}^{2}, b \cdot P=0, \hat{\zeta}, \eta v \cdot P\right) / \widetilde{\mathscr{S}}\left(b^{2}, \ldots\right) \\
\tilde{f}_{1 T}^{\perp[1](1)}\left(b_{T}^{2}, \hat{\zeta}, \ldots, \eta v \cdot P\right) & =-2 \widetilde{A}_{12 B}\left(-b_{T}^{2}, b \cdot P=0, \hat{\zeta}, \eta v \cdot P\right) / \widetilde{\mathscr{S}}\left(b^{2}, \ldots\right) \\
\tilde{h}_{1}^{\perp[1](1)}\left(b_{T}^{2}, \hat{\zeta}, \ldots, \eta v \cdot P\right) & =2 \widetilde{A}_{4 B}\left(-b_{T}^{2}, b \cdot P=0, \hat{\zeta}, \eta v \cdot P\right) / \widetilde{\mathscr{S}}\left(b^{2}, \ldots\right)
\end{aligned}
$$

where the generic Fourier-transformed TMD is defined as

$$
\tilde{f}^{[1](n)}\left(b_{T}^{2}, \ldots\right)=n !\left(-\frac{2}{m_{N}^{2}} \partial_{b_{T}^{2}}\right)^{n} \int_{-1}^{1} d x \int d^{2} k_{T} e^{i b_{T} \cdot k_{T}} f\left(x, k_{T}^{2}, \ldots\right) .
$$

Here, the superscript ${ }^{[1]}$ denotes the first Mellin moment in $x$. In (2.13), the $b_{T} \rightarrow 0$ limit formally yields $k_{T}$-moments of TMDs. However, this limit contains additional singularities, which one can view as being regulated by a finite $b_{T}$. In the following, results will only be given at finite $b_{T}$. Note the presence of the soft factors $\widetilde{\mathscr{S}}$ on the right-hand sides of (2.10)-(2.12). One can construct observables in which the soft factors cancel by normalizing the (Fourier-transformed) Sivers and Boer-Mulders functions (2.11) and (2.12) by the unpolarized TMD (2.10), which essentially counts the number of valence quarks. Thus, one defines the "generalized Sivers shift"

$$
\left\langle k_{y}\right\rangle_{T U}\left(b_{T}^{2}, \ldots\right) \equiv m_{N} \frac{\tilde{f}_{1 T}^{\perp[1](1)}\left(b_{T}^{2}, \ldots\right)}{\tilde{f}_{1}^{[1](0)}\left(b_{T}^{2}, \ldots\right)}=-m_{N} \frac{\widetilde{A}_{12 B}\left(-b_{T}^{2}, 0, \hat{\zeta}, \eta v \cdot P\right)}{\widetilde{A}_{2 B}\left(-b_{T}^{2}, 0, \hat{\zeta}, \eta v \cdot P\right)}
$$

which is the regularized, finite- $b_{T}$ generalization of the "Sivers shift"

$$
m_{N} \frac{\tilde{f}_{1 T}^{\perp[1](1)}(0, \ldots)}{\tilde{f}_{1}^{[1](0)}(0, \ldots)}=\frac{\int d x \int d^{2} k_{T} k_{y} \Phi^{\left[\gamma^{+}\right]}\left(x, k_{T}, S_{T}=(1,0)\right)}{\int d x \int d^{2} k_{T} \Phi^{\left[\gamma^{+}\right]}\left(x, k_{T}, S_{T}=(1,0)\right)},
$$




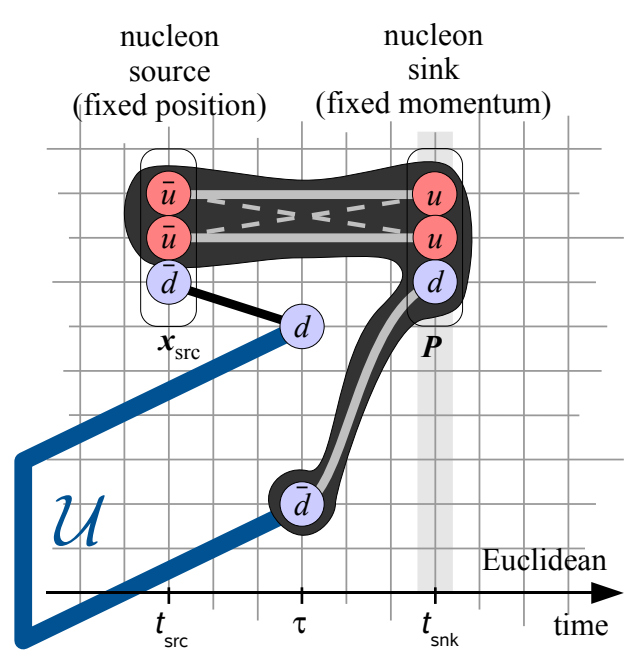

Figure 3: Lattice QCD scheme for evaluating matrix elements of the type (2.2) in a nucleon state, cf. main text. The staple-shaped gauge connection $\mathscr{U}$ is defined at a single Euclidean time, the operator insertion time $\tau$; the thick blue path representing $\mathscr{U}$ should thus be viewed as protruding out from the plane of the page at the fixed time $\tau$. Shown is specifically the $d$-quark part of the matrix element.

which, in view of the right-hand side, formally represents the average transverse momentum of unpolarized (" $U$ ") quarks orthogonal to the transverse (" $T$ ") spin of the nucleon, normalized to the corresponding number of valence quarks. In the interpretation of (2.15), it should be noted that the numerator sums over the contributions from quarks and antiquarks, whereas the denominator contains the difference between quark and antiquark contributions, thus giving the number of valence quarks. Analogously, one can also extract the generalized Boer-Mulders shift

$$
\left\langle k_{y}\right\rangle_{U T}\left(b_{T}^{2}, \ldots\right)=m_{N} \frac{\widetilde{A}_{4 B}\left(-b_{T}^{2}, 0, \hat{\zeta}, \eta v \cdot P\right)}{\widetilde{A}_{2 B}\left(-b_{T}^{2}, 0, \hat{\zeta}, \eta v \cdot P\right)} .
$$

Note that the ratios (2.14) and (2.16), besides canceling soft factors, also cancel $\Gamma$-independent multiplicative wave function renormalization constants attached to the quark operators in (2.2) at finite physical separation $b$.

\section{Lattice evaluation}

The formal framework laid out above provides all the necessary elements for a lattice QCD evaluation of generalized shifts such as (2.14) and (2.16). The path towards these observables proceeds via the calculation of nucleon matrix elements of the type (2.2) and subsequent decomposition into invariant amplitudes, as given in (2.7)-(2.9). To elucidate the strictures imposed on the calculational framework by employing lattice QCD to determine the nucleon matrix elements (2.2), it is useful to briefly review the standard scheme used to evaluate such matrix elements. As depicted in Fig. 3, one places a source and a sink with the quantum numbers of the nucleon 
state under consideration ${ }^{1}$ at locations on the Euclidean lattice widely separated in Euclidean, i.e., imaginary time; then, imaginary time evolution generates a bona fide nucleon ground state at intermediate times well separated from source and sink due to exponential decay of excited state contributions. In this region, one can evaluate nucleon ground state matrix elements. However, this computational setup implies a strong restriction on the type of matrix element that can be evaluated. Having already used the temporal direction on the lattice to represent an imaginary time coordinate, one cannot represent Minkowski, i.e., real time separations on the lattice. Consequently, only the matrix elements of operators which are defined at a single time can be straightforwardly accessed in this standard scheme.

This is the point where it becomes crucial to be working with a definition of the fundamental TMD correlator (2.2) in which the four-vectors $b$ and $v$ are generically space-like. Only in that case is there no obstacle to boosting the problem from the Lorentz frame in which (2.2) is originally defined to a frame in which $b$ and $v$ are purely spatial, and evaluating $\widetilde{\Phi}_{\text {unsubtr. }}^{[\Gamma]}$ in that frame using lattice QCD. Having accomplished that, it is the decomposition into invariant amplitudes (2.7)(2.9) which permits the connection back to the original Lorentz frame; the results extracted for the invariant amplitudes $\widetilde{A}_{i B}$ are immediately valid also in that frame, thus completing the determination of quantities of the type (2.14) and (2.16).

One special aspect of the lattice TMD calculation pursued here is the large number of matrix element evaluations implied by a survey of staple-shaped link geometries ${ }^{2}$ characterized in terms of the separations $b$ and $\eta v$. This is efficiently handled by the sequential propagator technique: As depicted in Fig. 3, one evaluates the forward quark propagator, which not only provides the propagation of the $d$-quark from source to operator insertion (black line in Fig. 3), but two of which can furthermore be contracted at the sink time $t_{\text {snk }}$ to generate a new source for subsequent propagation from $t_{\text {snk }}$ back to the operator insertion time $\tau$. Note that the contraction at $t_{\text {snk }}$ also includes the projection onto the desired nucleon three-momentum $\mathbf{P}$. The sequential propagator (dark shaded region in Fig. 3) determined in this manner, combined with the forward propagator, thus only has to be calculated once for given $\mathbf{P}$ and then, finally, can be contracted with a large set of gauge connections $\mathscr{U}$ described by different $b$ and $\eta v$ to arrive at the corresponding set of matrix elements.

Since, in a numerical lattice calculation, the staple extent $\eta$ necessarily remains finite, two extrapolations must be performed from the generated data, namely, the one to infinite staple length, $\eta \rightarrow \pm \infty$, and the extrapolation of the staple direction towards the light cone, $\hat{\zeta} \rightarrow \infty$. As shown below, the former extrapolation is under control for a range of parameters used in this work, whereas the latter extrapolation presents a formidable challenge. The main limitation in this respect is the set of nucleon three-momenta $\mathbf{P}$ accessible with sufficient statistical accuracy; limited $\mathbf{P}$ implies limited $\hat{\zeta}$, cf. (2.6). In the following, only data for the isovector, $u-d$ quark combination will be

\footnotetext{
${ }^{1}$ In practice, projection onto definite three-momentum $\mathbf{P}$ is only performed at the sink; together with projection onto zero momentum transfer at the operator insertion, momentum conservation then implies that also only the source component with momentum $\mathbf{P}$ contributes to the matrix element.

${ }^{2}$ The set of relevant geometries is subject to certain constraints, which however only partially mitigate the volume of the calculation: Concentrating on the lowest $x$-moments, cf. (2.13), implies evaluation specifically at $b \cdot P=0$. Together with $b^{+}=0$, this means that $b$ is purely transverse. On the other hand, in the adopted framework [5,6], $v$ is taken to have no transverse components, and, therefore, also $b \cdot v=0$.
} 

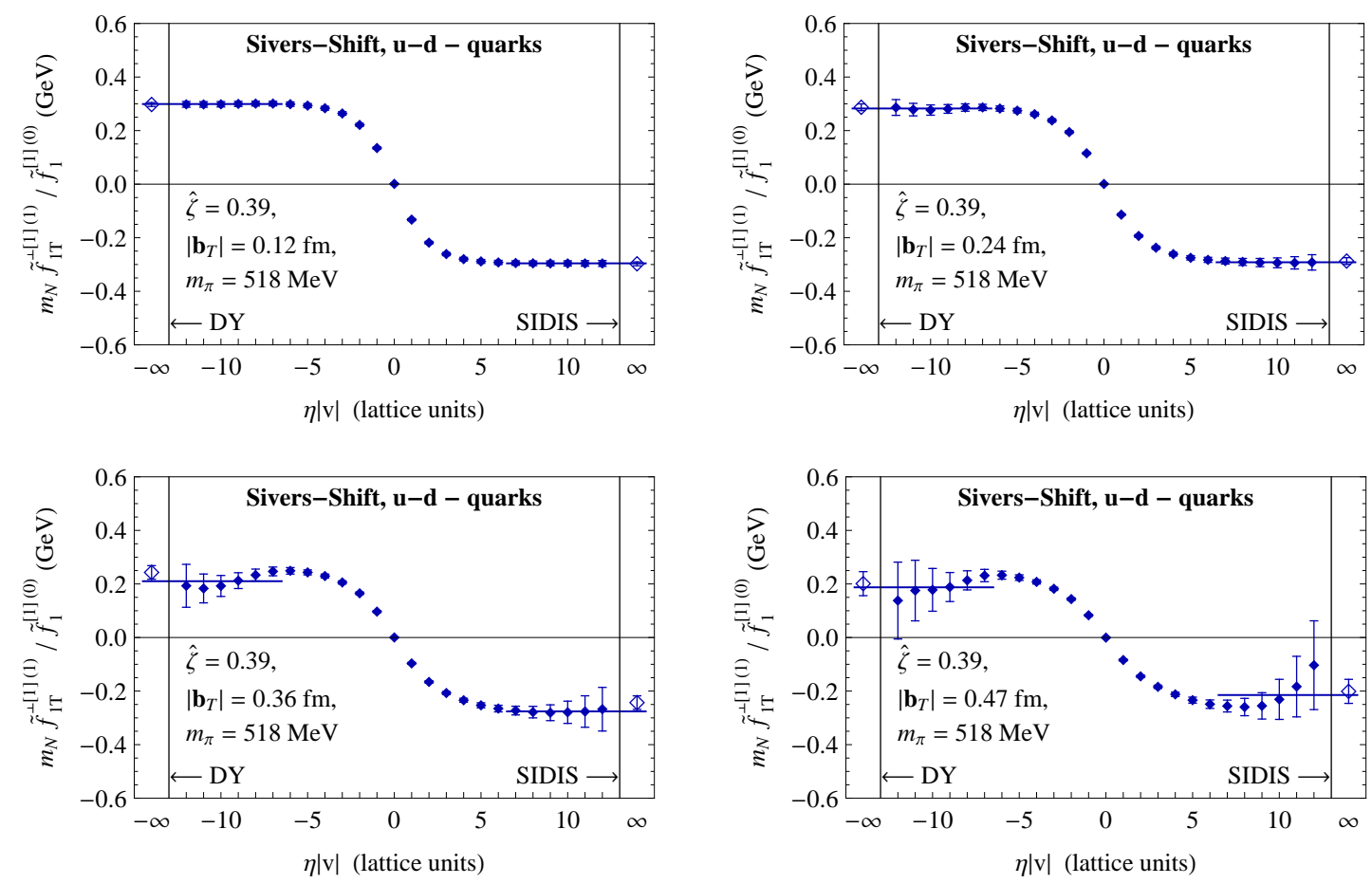

Figure 4: Generalized Sivers shift as a function of the staple extent $\eta|v|$, with the different panels showing a sequence of results obtained for increasing quark separation $b_{T}$. The parameter $\hat{\zeta}$ characterizing the approach to the light cone is fixed; from [9].

shown, since in this channel, couplings of the operator insertion to disconnected quark loops in the nucleon cancel. Such disconnected contributions have not been evaluated. Calculations were performed on three MILC 2+1-flavor gauge ensembles [11] with a lattice spacing of $a=0.12 \mathrm{fm}$, corresponding to pion masses $m_{\pi}=369 \mathrm{MeV}$ and $m_{\pi}=518 \mathrm{MeV}$, with two lattice sizes used in the former case, $20^{3} \times 64$ and $28^{3} \times 64$. For $m_{\pi}=518 \mathrm{MeV}$, the lattice size is $20^{3} \times 64$. In addition to the three-momenta $\mathbf{P}=0$ and $\mathbf{P}=(-1,0,0) \cdot 2 \pi / L$ (where $L$ denotes the spatial lattice extent), which were employed on all ensembles, in the heavier pion mass ensemble, fraught with less statistical uncertainty, also $\mathbf{P}=(1,-1,0) \cdot 2 \pi / L$ and $\mathbf{P}=(-2,0,0) \cdot 2 \pi / L$ were used. The latter case, paired with $\mathbf{v}=( \pm 1,0,0)$, provides the largest $|\hat{\zeta}|$ value accessed, namely, $|\hat{\zeta}|=0.78$.

\section{Numerical results}

Figure 4 shows a sequence of data for the generalized Sivers shift ${ }^{3}(2.14)$ as a function of the staple extent $\eta|v|$, with the quark separation $b_{T}$ varying from panel to panel. The T-odd behavior of this observable, manifested in the antisymmetry in the variable $\eta|v|$, is evident, with $\eta \rightarrow \infty$ corresponding to the SIDIS limit, whereas $\eta \rightarrow-\infty$ yields the DY limit. The data level off to approach clearly identifiable, stable plateaux as the staple length grows. The limiting SIDIS and DY values, represented by the open symbols, are extracted by imposing antisymmetry in $\eta$, allowing

\footnotetext{
${ }^{3}$ For $m_{N}$, the value of the nucleon mass as determined on the ensemble under consideration is used, rather than the physical nucleon mass.
} 


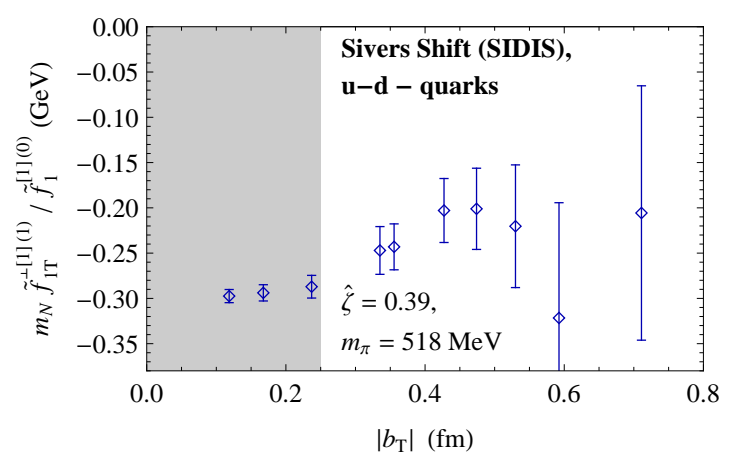

Figure 5: Dependence of the generalized Sivers shift on the quark separation $\left|b_{T}\right|$ in the $\eta \rightarrow \infty$ SIDIS limit; from [9].

one to appropriately average the $\eta \rightarrow \pm \infty$ plateau values. As the quark separation $b_{T}$ increases, statistical fluctuations become more noticeable, thus limiting the range of $b_{T}$ for which the plateaux yielding the SIDIS and DY limits can be extracted.

Fig. 5 summarizes the results obtained for the SIDIS limit as a function of $b_{T}$, at the same fixed $\hat{\zeta}$ as used in Fig. 4. The shaded area below $\left|b_{T}\right| \approx 0.25 \mathrm{fm}$ indicates the region where the results may be significantly affected by finite lattice cutoff effects as the quark operators in (2.2) approach one another. Thus, for $\hat{\zeta}=0.39$ and a limited range of $b_{T}$, a sizeable negative SIDIS Sivers shift is found in the isovector, $u-d$ quark case.

Turning to the dependence of the generalized Sivers shift on the Collins-Soper parameter $\hat{\zeta}$, Fig. 6 displays data for this shift as a function of the staple extent $\eta|v|$ analogous to Fig. 4, except that the panels correspond to varying $\hat{\zeta}$, while the quark separation $b_{T}$ is fixed at $\left|b_{T}\right|=0.36 \mathrm{fm}$. Thus, the lower left-hand panel in Fig. 4 fits in between the two panels shown in Fig. 6 in terms of a sequence of panels at different $\hat{\zeta}$ for a fixed $\left|b_{T}\right|$. Note that the $\hat{\zeta}=0$ data were obtained using $\mathbf{P}=0$, in which case one cannot identify a "forward" or a "backward" direction 4 . For this reason, only a single branch in $\eta|v|$ is displayed, the sign being a matter of definition. These data illustrate how the quality of the signal deteriorates as the nucleon momentum $|\mathbf{P}|$, and therefore
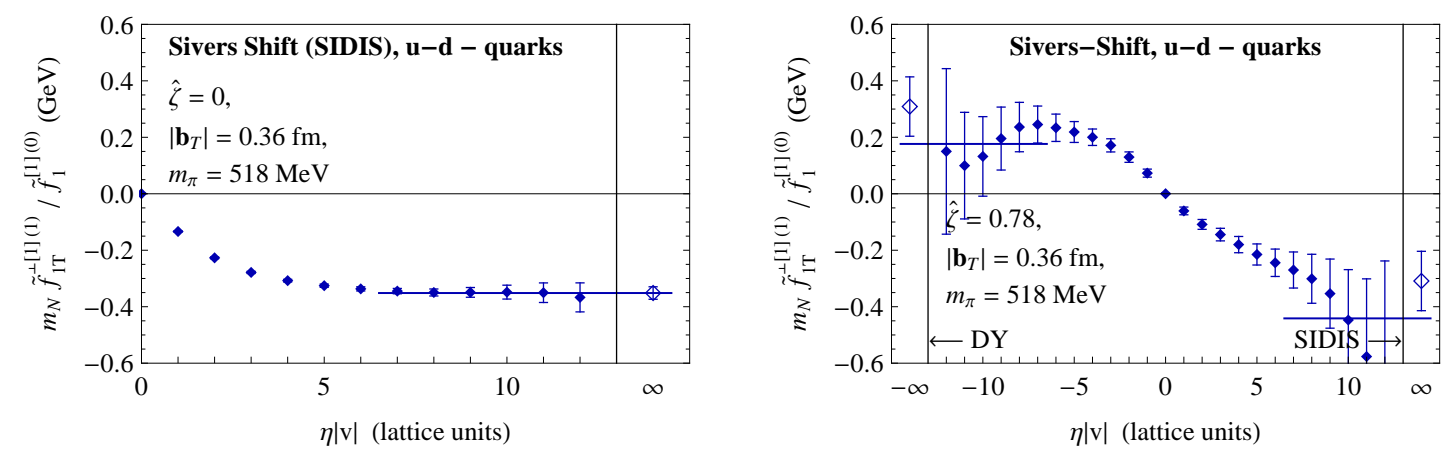

Figure 6: Generalized Sivers shift as a function of the staple extent $\eta|v|$, for two extreme values of the Collins-Soper parameter $\hat{\zeta}$; from [9].

\footnotetext{
${ }^{4}$ A way to define this limit cleanly is given in [9].
} 


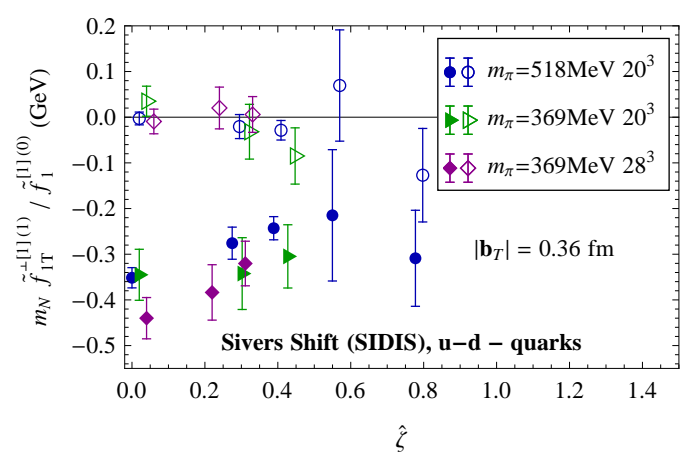

Figure 7: Dependence of the generalized Sivers shift on the Collins-Soper parameter $\hat{\zeta}$ in the $\eta \rightarrow \infty$ SIDIS limit for all three ensembles investigated. The quark separation $b_{T}$ is held fixed; from [9].

$\hat{\zeta}$, is increased, even when the same staple parameters $b$ and $\eta|v|$ are employed. At the largest Collins-Soper parameter accessed in this investigation, $\hat{\zeta}=0.78$, identification of plateaux in $\eta|v|$ becomes difficult, especially in the SIDIS direction. The plateau averages in the right-hand panel of Fig. 6 were formed using the same range of $\eta|v|$ as in all other cases considered.

Fig. 7 summarizes the SIDIS limit results as a function of $\hat{\zeta}$ for the same $\left|b_{T}\right|=0.36 \mathrm{fm}$ as used in Fig. 6, with the $m_{\pi}=518 \mathrm{MeV}$ data discussed up to this point supplemented by results from the other two ensembles included in this investigation. Note that the relevant data are represented by the full symbols; the empty symbols correspond to a certain partial contribution to the Sivers shift which will not be discussed further here; for details, cf. [9]. The statistical uncertainty of the generalized Sivers shift quickly increases as $\hat{\zeta}$ is raised. No clear trend as a function of $\hat{\zeta}$ can be identified at the present level of accuracy, and connecting with perturbative evolution equations at large $\hat{\zeta}$ will clearly represent the most difficult challenge for the present approach. Furthermore, within the (sizeable) uncertainties, no significant variation of the generalized Sivers shift can be discerned as one changes the pion mass or the spatial extent of the lattice.

The generalized Boer-Mulders shift (2.16) exhibits characteristics similar to the ones of the generalized Sivers shift. Fig. 8 shows representative results for the dependence on the staple extent
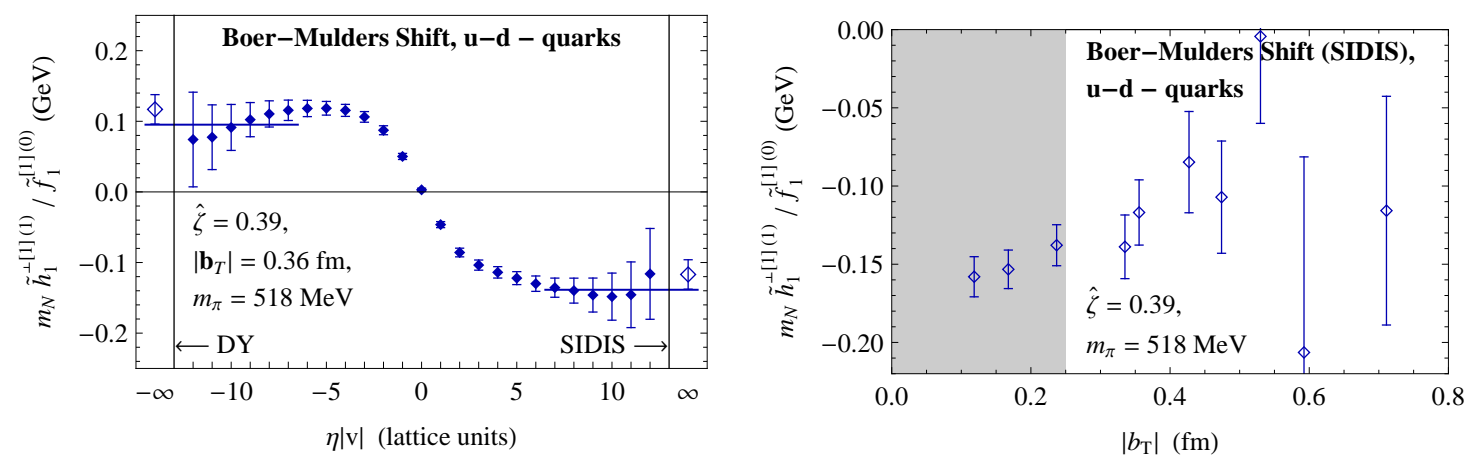

Figure 8: Results for generalized Boer-Mulders shift. Left: Dependence on the staple extent $\eta|v|$ for fixed quark separation $b_{T}$ and Collins-Soper parameter $\hat{\zeta}$; right: Results in the $\eta \rightarrow \infty$ SIDIS limit as a function of $b_{T}$ for fixed $\hat{\zeta}$. From [9]. 


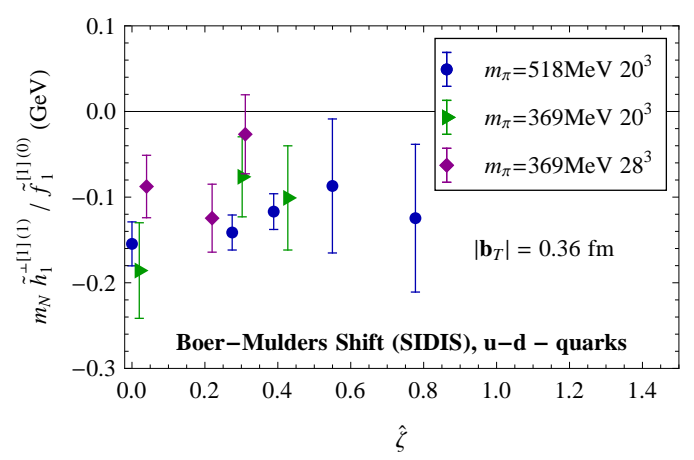

Figure 9: Results for generalized Boer-Mulders shift as a function of $\hat{\zeta}$ for all ensembles; from [9].

$\eta|v|$ and the dependence of the $\eta \rightarrow \infty$ SIDIS limit result on the quark separation $b_{T}$. The T-odd behavior of the observable is again evident, and plateaux can be identified as the staple length grows. The SIDIS limit data as a function of $b_{T}$ closely parallel the behavior of the generalized Sivers shift displayed in Fig. 5. The dependence of the generalized Boer-Mulders shift in the SIDIS limit on the Collins-Soper evolution parameter $\hat{\zeta}$, for all three ensembles considered in this investigation, is summarized in Fig. 9 for a fixed quark separation $\left|b_{T}\right|$. Again, the statistical uncertainty of the observable quickly increases as $\hat{\zeta}$ is raised, precluding the identification of any trend as a function of $\hat{\zeta}$ which might aid in connecting the results with perturbative evolution equations at large $\hat{\zeta}$. Within the sizeable uncertainties, the data obtained at different pion masses and spatial lattice extents are compatible with one another.

Comparing the results for the Sivers and Boer-Mulders shifts in the isovector, $u-d$ flavor channel considered above, the magnitude of the Boer-Mulders shift is smaller than the one of its Sivers counterpart, and the signal for the former is of less high quality than the one for the latter. One reason for this is that, if one separates the $u$-and $d$-quark contributions, the Sivers shifts in the two cases are of opposite sign (thus reinforcing each other in the $u-d$ difference), whereas the Boer-Mulders shifts are of the same sign, thus canceling each other to some extent.

It should be remarked that the qualitative observations on the behavior of the Sivers and BoerMulders shifts made here are compatible with phenomenological analyses of experimental SIDIS data [12,13], as well as considerations based on the chromodynamic lensing mechanism $[14,15]$. However, it must be stressed that a variety of systematic effects still need to be taken into account before a fully quantitative comparison can be envisaged.

\section{Summary and outlook}

This exploratory study of TMDs within lattice QCD, employing staple-shaped gauge connections to incorporate final/initial state effects (for SIDIS/DY), has provided first results for T-odd Sivers and Boer-Mulders observables ${ }^{5}$. Both of the corresponding TMDs are sizeable and negative in the isovector, $u-d$ quark case. To cancel soft factors and multiplicative renormalization constants, appropriate ratios of Fourier-transformed TMDs ("generalized shifts", cf. (2.14) and (2.16))

\footnotetext{
${ }^{5}$ Complementary results on T-even TMDs are also available and are presented in the comprehensive report [9].
} 
were constructed. The staple direction $v$ was taken to be generically space-like, with the light-cone limit to be approached by extrapolation in the Collins-Soper parameter $\hat{\zeta}$. This extrapolation has to be performed in addition to the one to infinite staple extents $\eta$. While the latter extrapolation is under control for a range of parameters considered in this work, the limit $\hat{\zeta} \rightarrow \infty$ clearly presents a formidable challenge for the approach presented here. With this in mind, the Boer-Mulders function of the pion is presently being investigated. Both the lower mass of the pion compared with the one of the nucleon (note that the hadron mass enters the denominator of $\hat{\zeta}$ in (2.6)), as well as the reduced statistical fluctuations of pion correlators, permitting the treatment of higher hadron momenta, are expected to aid in accessing lattice data at higher $\hat{\zeta}$.

\section{Acknowledgments}

The lattice calculations performed in this work relied on the Chroma software suite [16] and employed computing resources provided by the U.S. DOE through USQCD at Jefferson Lab. Support by the Heisenberg-Fellowship program of the DFG (P.H.), SFB/TRR-55 (A.S.), and the U.S. DOE through grants DE-FG02-96ER40965 (M.E.) and DE-FG02-94ER40818 (J.N.), as well as through contract DE-AC05-06OR23177, under which Jefferson Science Associates, LLC, operates Jefferson Laboratory (B.M.), is acknowledged. M.E. furthermore is grateful to the Jefferson Lab Theory Center for its generous support and hospitality during the 2011/12 academic year, which proved invaluable for the progress of this project.

\section{References}

[1] D. Boer, M. Diehl, R. Milner, R. Venugopalan, W. Vogelsang, et al., arXiv:1108.1713.

[2] M. Alekseev, et al., COMPASS Collaboration, Phys. Lett. B673 (2009) 127.

[3] A. Airapetian, et al., HERMES Collaboration, Phys. Rev. Lett. 103 (2009) 152002.

[4] H. Avakian, et al., CLAS Collaboration, Phys. Rev. Lett. 105 (2010) 262002.

[5] S. M. Aybat and T. C. Rogers, Phys. Rev. D 83 (2011) 114042.

[6] J. C. Collins, Foundations of Perturbative QCD (Cambridge University Press, 2011).

[7] S. M. Aybat, J. C. Collins, J.-W. Qiu and T. C. Rogers, Phys. Rev. D 85 (2012) 034043.

[8] J. C. Collins and T. C. Rogers, arXiv:1210.2100.

[9] B. Musch, P. Hägler, M. Engelhardt, J. W. Negele and A. Schäfer, Phys. Rev. D 85 (2012) 094510.

[10] T. C. Rogers and P. J. Mulders, Phys. Rev. D 81 (2010) 094006.

[11] C. Aubin, C. Bernard, C. DeTar, J. Osborn, S. Gottlieb, E. Gregory, D. Toussaint, U. Heller, J. Hetrick and R. Sugar, Phys. Rev. D 70 (2004) 094505.

[12] M. Anselmino, M. Boglione, U. D’ Alesio, S. Melis, F. Murgia and A. Prokudin, arXiv: 1107.4446.

[13] V. Barone, S. Melis and A. Prokudin, Phys. Rev. D 81 (2010) 114026.

[14] M. Burkardt, Nucl. Phys. A735 (2004) 185.

[15] M. Burkardt and B. Hannafious, Phys. Lett. B658 (2008) 130.

[16] R. G. Edwards and B. Joó, SciDAC Collaboration, Nucl. Phys. Proc. Suppl. 140 (2005) 832. 